\title{
Response to 'Preserved corticospinal conduction without voluntary movement after spinal cord injury'
}

\author{
Spinal Cord (2014) 52, 717; doi:10.1038/sc.2014.115; published online 22 July 2014
}

We read with interest the article by Edwards et al. ${ }^{1}$ In a subject with a 6-year history of traumatic spinal cord injury (C5; AIS 'B'), these authors reported normal motor-evoked potentials (MEPs) from wrist extensors in the absence of significant voluntary wrist extension (1/5 motor power). This is interpreted as preservation of corticospinal tract function, without corresponding voluntary function, reminiscent of Milan Dimitrijevic's 'discomplete' story. ${ }^{2}$

We believe a more plausible explanation of the finding from Edwards et al. can be attributed to their use of surface-recording electrodes, and the probability of relatively close proximity of 'ECR'recording electrodes to the brachioradialis. This muscle receives strong innervation from the C5/6 root, and in persons with SCI it typically shows a functional preservation pattern paralleling that of biceps brachii.

Volume conduction from the brachioradialis would explain the weak EMG in both wrist flexors and extensors during attempted movements in these directions. Had the investigators asked the subject to flex the elbow with the forearm mid-way between pronation and supination, we believe a larger interference pattern from both wrist flexors and (especially) extensors would have been evident than was seen with wrist flexion/extension attempts.
When assessing voluntary contraction preservation using MEPs, it is easy to be fooled by this forearm muscle. One has to be especially careful when suggesting that normal MEPs can be present in a given muscle group in the absence of voluntary movement. In our experience in this area, both in the operating room and in the lab, we have only seen this in conversion disorder (hysterical paralysis), and we know the problems there lie well rostral to the spinal cord!

\section{CONFLICT OF INTEREST}

The authors declare no conflict of interest.

B Calancie and N Alexeeva Department of Neurosurgery, Upstate Medical University, Syracuse, NY, USA E-mail: calancib@upstate.edu

1 Edwards DJ, Cortes M, Thickbroom GW, Rykman A, Pascual-Leone A, Volpe BT. Preserved corticospinal conduction without voluntary movement after spinal cord injury. Spinal Cord 2013; 51: 765-767.

2 Sherwood AM, Dimitrijevic MR, McKay WB. Evidence of subclinical brain influence in clinically complete spinal cord injury: discomplete SCI. J Neurol Sci 1985; 110: 90-98. 